\title{
Fault Diagnosis of Power Transformer based on EMD Technology
}

\author{
Wenliang Peng1, 2, a, En Zhang 2, b, Guangrong Bian ${ }^{3, c}$ and HuiYuan Zhao ${ }^{2, d}$ \\ ${ }^{1}$ Wuhan University of Technology, Wuhan430061, China; \\ 2Zhuhai Campus Beijing Institute of Technology, Zhuhai, 519080, China. \\ ${ }^{3}$ Department of Aviation Ammunition, Air Force Service College, Xuzhou221000, China \\ a85178005@qq.com, b18942596@126.com, ‘44973901@qq.com, d236862828@qq.com
}

Keywords: EMD, transformer, fault diagnosis.

\begin{abstract}
This paper focuses on the use of EMD technology to transformer fault diagnosis. Through continuous analysis and in-depth study on large amounts of data, according to the experience and the related algorithms are derived for intrinsic mode function, this method is the EMD algorithm, namely EMD algorithm, its core algorithm is HHT transform. By this method, the data is too large and difficult to classify and analyze the case, get better intrinsic mode function (Intrinsic Mode Function, referred to as IMF). The data were analysed by IMF, can draw the fault types of transformer under different conditions.
\end{abstract}

\section{Introduction}

The technology of EMD is to decompose the empirical model, decompose a large number of complex data signals into a number of eigenmode functions, and these decomposed IMF components with the original signal of different time scales of local features signal ${ }^{[1]}$. In this way, the unstable data is converted into stationary data, and the processed data is not distorted, and then the Hilbert transform method is used to form the time spectrum and has a certain frequency, thus forming an adaptive Features ${ }^{[2]}$.

\section{IMF Algorithm Steps}

As we all know, any signal we can use the plural form to express, the plural includes the real and imaginary parts. In the EMD algorithm, we form a new series of steps to obtain a new time-frequency spectrum, using the following way to obtain the intrinsic mode function:

(1) First of all, we will measure the signal $\mathrm{x}(\mathrm{t})$ data for simple classification, to find its maximum value and the minimum value, the use of interpolation algorithm and cubic spline function were formed on the original sequence of the upper and lower envelope, Where $U p(t)$ is the upper envelope and Down ( $\mathrm{t}$ ) is the lower envelope. Up and down Avg (t) is the mean of the upper and lower envelopes, the formula is ${ }^{[3]}$ :

$\operatorname{Avg}(t)=\frac{U p(t)+\operatorname{Down}(t)}{2}$

(2) with the original signal $\mathrm{x}(\mathrm{t})$ and the difference between the mean can be a new signal New $(\mathrm{t})$ : $\operatorname{New}(t)=x(t)-\operatorname{Avg}(t)$

The new signal $\mathrm{x}(\mathrm{t})$ is not the IMF because it is unstable and does not meet the IMF's characteristics. Therefore, we must repeat the steps of (2), in general, no more than 10 times we can get the first eigenmode function component $\mathrm{C}_{1}(\mathrm{t})$ we want, assuming that the signal obtained after $\mathrm{k}$ times is Newk (t), We define ${ }^{[4]}$ :

$C_{1}(t)=\operatorname{imf}_{1}(t)=\mathrm{NeW}^{k}(t)$

(3) subtracting the original IMF component $\mathrm{C}_{1}(\mathrm{t})$ from the original signal $\mathrm{x}(\mathrm{t})$, we can get the second eigenfunction function component $\mathrm{C}_{2}(\mathrm{t})$, the formula is:

$C_{2}(t)=x(t)-C_{1}(t)$ 
Repeat (3) this step, assuming that after $\mathrm{n}$ times, $\mathrm{Cn}(\mathrm{t})$ satisfies its residual value $\mathrm{Rn}(\mathrm{t})$ is less than a predetermined value or $\mathrm{Rn}(\mathrm{t})$ is a constant or monotonic function, it is not necessary to repeat. So the original signal $\mathrm{x}(\mathrm{t})$ after EMD algorithm processing, you can get:

$$
x(t)=\sum_{i=1}^{n} C_{i}(t)+R_{n}(t)
$$

Where $\mathrm{Ci}(\mathrm{t})$ is the $\mathrm{i}$-th IMF component (the $\mathrm{i}$-th decomposition) and $\mathrm{Rn}(\mathrm{t})$ is the residual value.

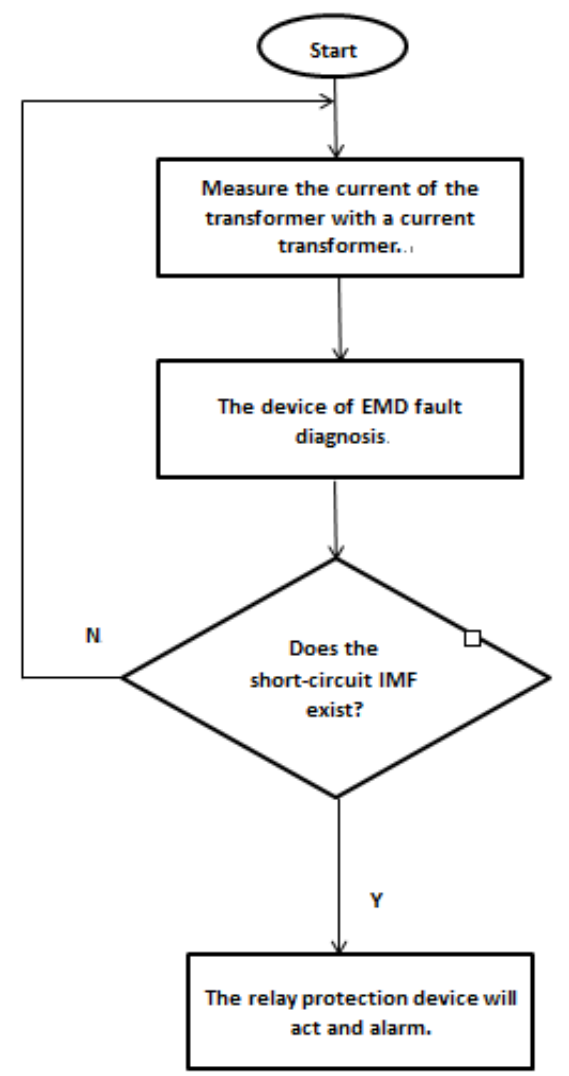

Fig.1 The flow chart of fault diagnosis

The analysis method of transformer fault diagnosis method based on EMD technology is as follows in this chapter:

(1) The current signal and gas signal of transformer are obtained from the monitoring system. The signals can be measured by current transformers, TA and gas sensors, which are collected by the SCADA system and classified briefly ${ }^{[5]}$.

(2) The collected data is sent to the EMD fault detector for analysis.

(3) EMD principle is used to analyze the data signal by empirical mode decomposition to obtain IMF.

(4) The IMF signal is transformed by Hilbert method, and the time and frequency spectrum of the analysis signal is compared with the original predetermined value to judge the fault, type and damage degree of the transformer.

(5) If the fault is serious, start the relay and turn off the power to protect it.

\section{Analysis}

We analyzed the data of transformer operation in a 220KV substation in Zhuhai. The experimental object is the SSZ10-K-180000/220 type three-phase transformer produced by Jiangsu Hua Peng Transformer Co., Ltd. the rated voltage is $220 / 110 / 10.5 \mathrm{KV}$, and the connection group number is YNyn0d11. We have installed 6 current transformers on this transformer and 6 voltage transformers, to measure current and voltage values flowing in and out of them.

The following is the data provided by the power supply bureau: at 9:15 on September 7, 2015, the bridge and bridge to change 513 calcium carbide plant line over-current I section protection action, 
switch tripping, circuit current 5.958kA. In September 9th 8:50, bridge 313 often Xinyi line el3 action, switch tripping, short-circuit current of $6.6032 \mathrm{kA}$, when the low voltage side of main transformer in independent operation. According to the trip information, provide the basis for the parameters of the transformer manufacturers of Power Supply Bureau, 1\# main transformer voltage withstand short-circuit current capacity: 14.01kA, voltage withstand short-circuit current capacity: 19.6kA, compared with the actual short-circuit current in main transformer short circuit withstand current range. Fig. 2.3 is a frequency spectrum of the IMF signal of the high voltage winding extracted from the 2015.9.7 when the fault occurs, indicating that the high voltage winding has been out of order. However, the later stage diagram is back, and it can be concluded that the HV winding should be free of faults.

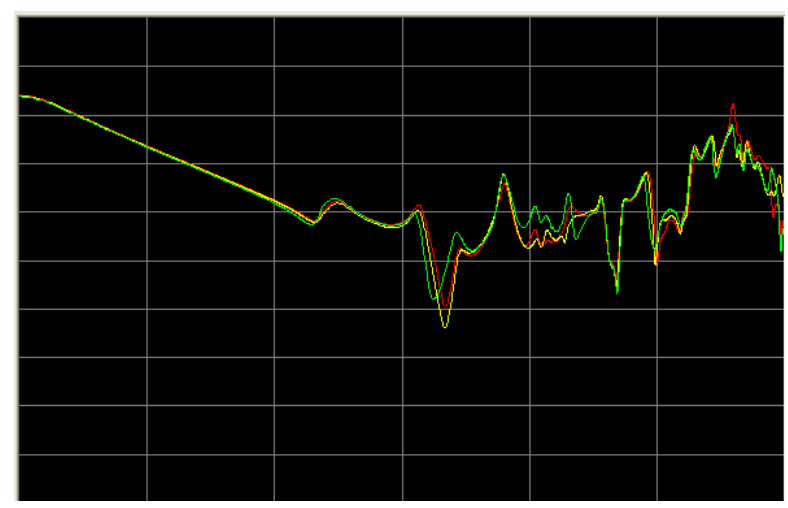

Fig.2 The figure of the IMF spectrum of high-voltage winding

It can be seen from the figure, if not the failure of the transformer, line should be a comparison of parallel lines, such as when a fault occurs, the unbalanced current in the transformer outlet, will appear as in Figure 2 right half part of the curve, if the change is too large, the situation is more serious. In the small fault transformer, curve spectrum in general is not great, can continue to run; if the outlet fast change situation, the emergence of a large or large transformer fault, must immediately stop check. Fig. 3 is a IMF spectrum of the medium voltage winding, indicating that the medium voltage winding is also out of order and that the current has increased rapidly. This time must be down to check the transformer, otherwise the transformer will be damaged, under normal circumstances, the staff will be in accordance with the relevant provisions, apply to their superiors after the start of downtime, security transformer can not be damaged.

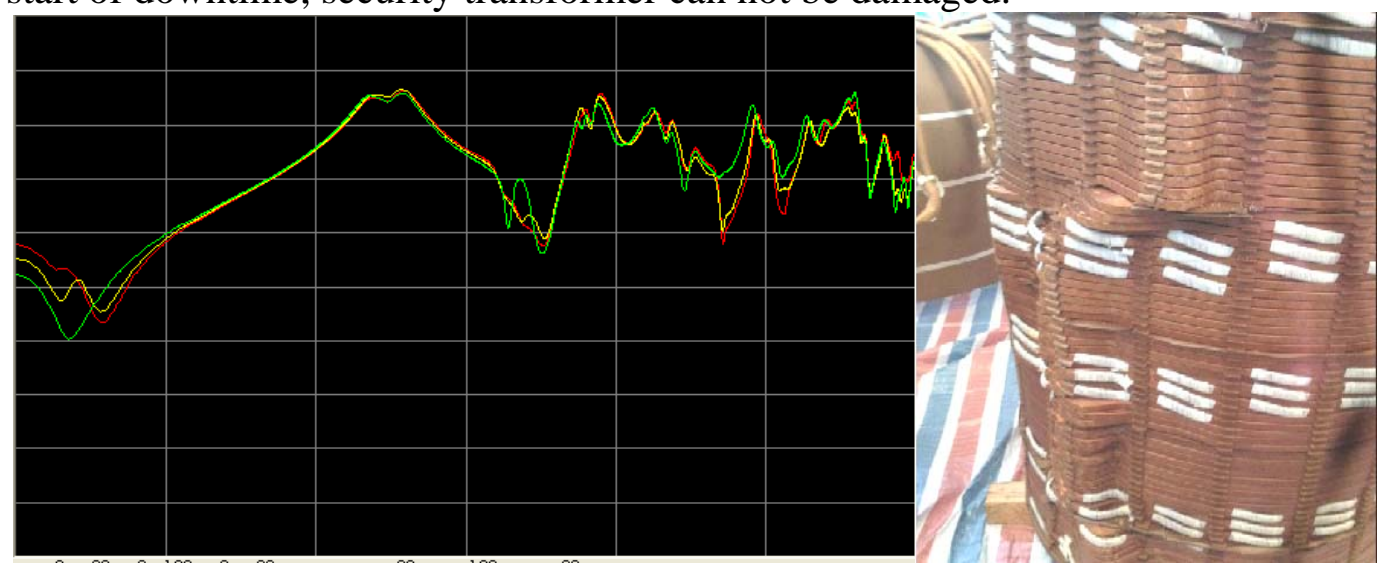

Fig.3 The figure of the IMF spectrum of medium-voltage winding 

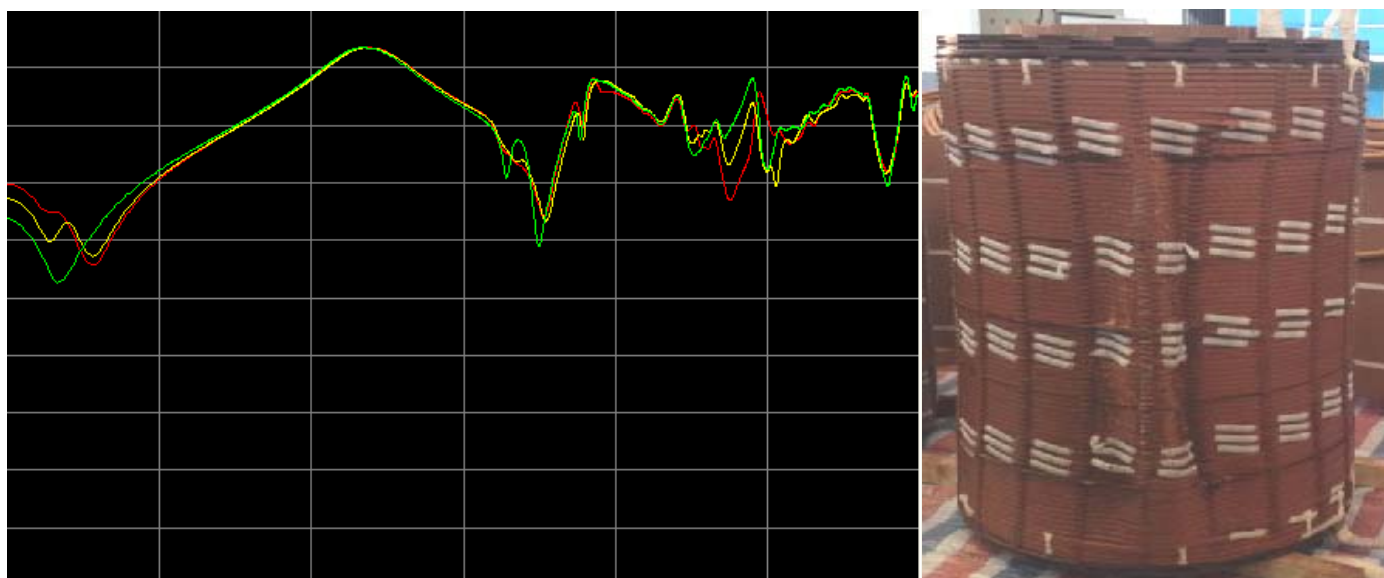

Fig.4 The figure of the IMF spectrum of low-voltage winding

Fig.4 is a IMF spectrum of the low voltage winding, indicating that the low-voltage winding also has a fault. From the high, medium and low voltage winding spectrum, we can see that this transformer has been out of order and must be checked out. The next 2 diagrams are the transformer windings removed.

It can be seen,low-voltage winding and medium voltage winding has obvious deformation, change the relative distribution of the capacitance caused by the high and low levels of winding, and low to high school and high school, on the capacitance low and there is a big change. At the same time, the low side winding has a slight deformation, which has a certain influence on the capacitance distribution. In particular the transformer outlet or near fault, short-circuit current of the transformer by huge electric power normal dozens of times even hundreds times, and the huge impact of current transformer winding vast sharply heated, winding mechanical strength greatly reduced, resulting in the winding deformation bulge, which is the main reason. At the same time, because of the frequent impact of large current in the $110 \mathrm{kV}$ middle voltage side, the mid voltage coil of the transformer is gradually unstable and generates accumulated deformation.

\section{Summary}

In short, through the EMD technology can more effectively diagnose the transformer fault, so that the transformer can be more stable work.

\section{References}

[1]. Mizutani Y, Takahashi T, Ito T. Lifetime evaluation method for pole transformer based on transienttemperature analysis [J]. IEEE Transactions on Power and Energy,2007,127(5): 653-658

[2]. M. García-Gracia, M. Villén, M.A. Cova, N. El Halabi. Detailed three-phase circuit model for power transformers over wide frequency range based on design parameters[J].Electric Power Systems Research, Volume 92, November 2012, Pages 115-122

[3]. D.V.S.Sarma, G.N.S. Kalyani. ANN aproach for condition monitoring of transformer using DGA[C]. TENCON, 2004 ' Vol.3, p444-447

[4]. E Ricci,G Zen,N Sebe,S Messelodi.A Prototype Learning Framework Using EMD: Application to Complex Scenes Analysis [J]. IEEE Transactions on Pattern Analysis \& Machine Intelligence, 2013, 35(3):513-526

[5]. Juan A. Martinez-Velasco. Equivalent circuit of transformers with control of voltage and phase angle [J]. Electric Power Systems Research, Volume 81, Issue 7, July 2011, Pages 1349-1356 